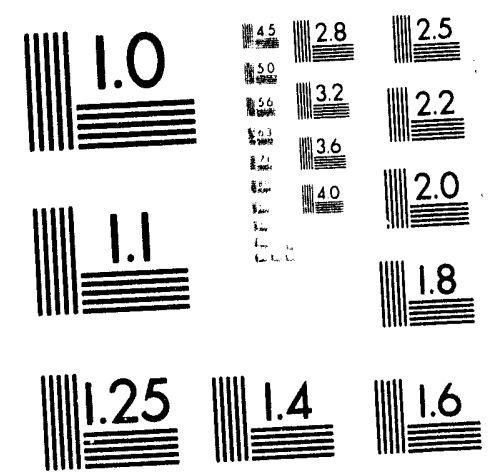



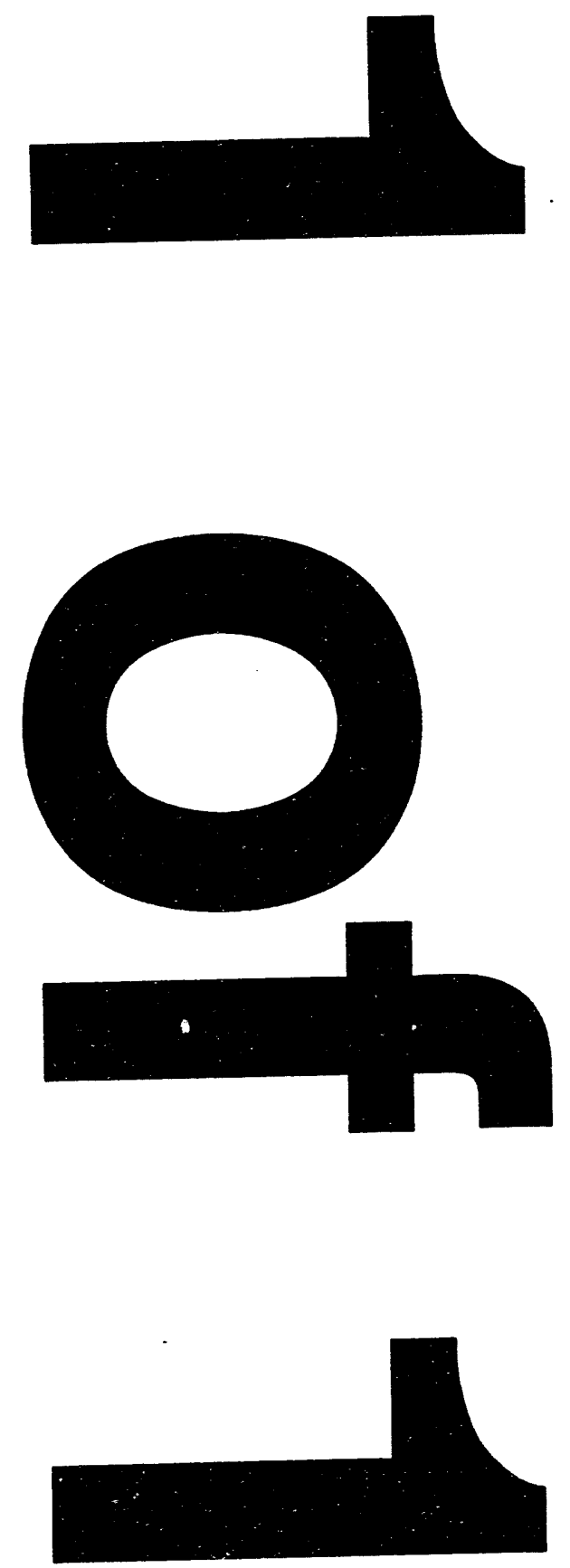


\title{
FUSION APPLICATIONS OF HIGH POWER MILLIMETER WAVE SOURCES
}

\author{
by \\ R.L. FREEMAN and T.V. GEORGE*
}

This is a preprint of a paper presented at the SPIE International Conference on Millimeter and Submillimeter Waves and Applications, January 10-14, 1994, San Diego, California, and to be printed in the Digest.

Work partially supported by U.S. Department of Energy Contract DE-AC03-89ER52153

*U.S. Department of Energy

GENERAL ATOMICS PROJECT 3469 JANUARY 1994

GENERAL ATOMICS 


\title{
Fusion applications of high power millimeter wave sources
}

\author{
R.L. Freeman \\ General Atomics, RF Physics and Technology Division \\ San Diego, CA 92186-9784 \\ T.V. George \\ U.S. Department of Energy, ER-531, Germantown \\ Washington, D.C. 20585
}

\begin{abstract}
Heating by means of high power electron cyclotron (EC) waves in the mm wavelength range is considered to be one of the most attractive approaches for heating fusion plasmas to the temperatures required to achieve ignition. EC waves have also been used to drive plasma current by using directional launch and to stabilize MHD instabilities in tokamak plasmas through localized heating or current drive. Experiments are planned on both JET and TFTR to measure the alpha particle distribution by scattering EC waves.
\end{abstract}

The effectiveness of EC waves in heating magnetically confined plasmas is well established. Power is strongly absorbed near the cyclotron frequency of the electrons or at low harmonics. In a tokamak magnetic confinement device, the main toroidal magnetic field falls off inversely with the major radius, so for a given wave frequency a resonance exists only along a vertical line at one major radius. Hence, the heating can be easily localized within the plasma; usually it is desirable to deposit the energy at the plasma center for the most effective heating, but some applications require off-axis deposition. In addition to its proven effectiveness for plasma heating, the high power density transmission and the simple propagation characteristics of EC waves make it a desirable heating choice for fusion.

Despite all of these favorable characteristics, EC heating has not been widely employed due to the lack of suitable millimeter wave sources. The present target of the world-wide source development is a $1 \mathrm{MW} \mathrm{cW}$ gyrotron at $160 \mathrm{GHz}$ for application to ITER, the next-step fusion device being designed as a four-party international collaboration between the United States, the European Community, Japan, and the Russian Federation. It is anticipated that at least 50 MW of total power will be required for ITER, so even higher unit power than $1 \mathrm{MW}$ is desirable to minimize system costs and to take full advantage of the high power density which is possible for EC systems. EC wave heating is presently considered to be the alternate to ion cyclotron resonant heating rather than the primary heating due to the present lack of suitable EC wave sources.

The application to fusion heating will be illustrated by a description of the DIII-D EC wave experiments with up to $2 \mathrm{MW}$ of $60 \mathrm{GHz}$ power from $10200 \mathrm{~kW}$ Varian gyrotrons. A description of the new DIII-D prototype system designed for $1 \mathrm{MW}$ gyrotrons at $110 \mathrm{GHz}$ is representative of a state-of-the-art in EC wave systems.

As an example of the diagnostic applications in the fusion program using millimeter waves, the planned alpha particle scattering experiment on TFTR will be described. 
Substantial progress has been made throughout the world in the gyrotron development activities over the last few years in raising the unit power, the pulse length, and the frequency. The ITER target gyrotron parameters seem achievable within a few years given the pace of the present world-wide development programs. The progress in these activities will be summarized.

The free electron laser (FEL) is an alternate approach to generation of high power millimeter waves. These approaches become more competitive at higher frequencies. A FEL at Law :ence Livermore National Laboratory produced pulses of $2 \mathrm{GW}$ for $20 \mathrm{nsec}$ at $140 \mathrm{GHz}$ for application to the MTX tokamak. Also, a collaborative effort is underway on a tunable IEL at the FOM in the Netherlands.

\section{ACKNOWLEDGMENT}

This is a report of work partially supported by the U.S. Department of Energy under Contract DE-AC03-89ER52153. 

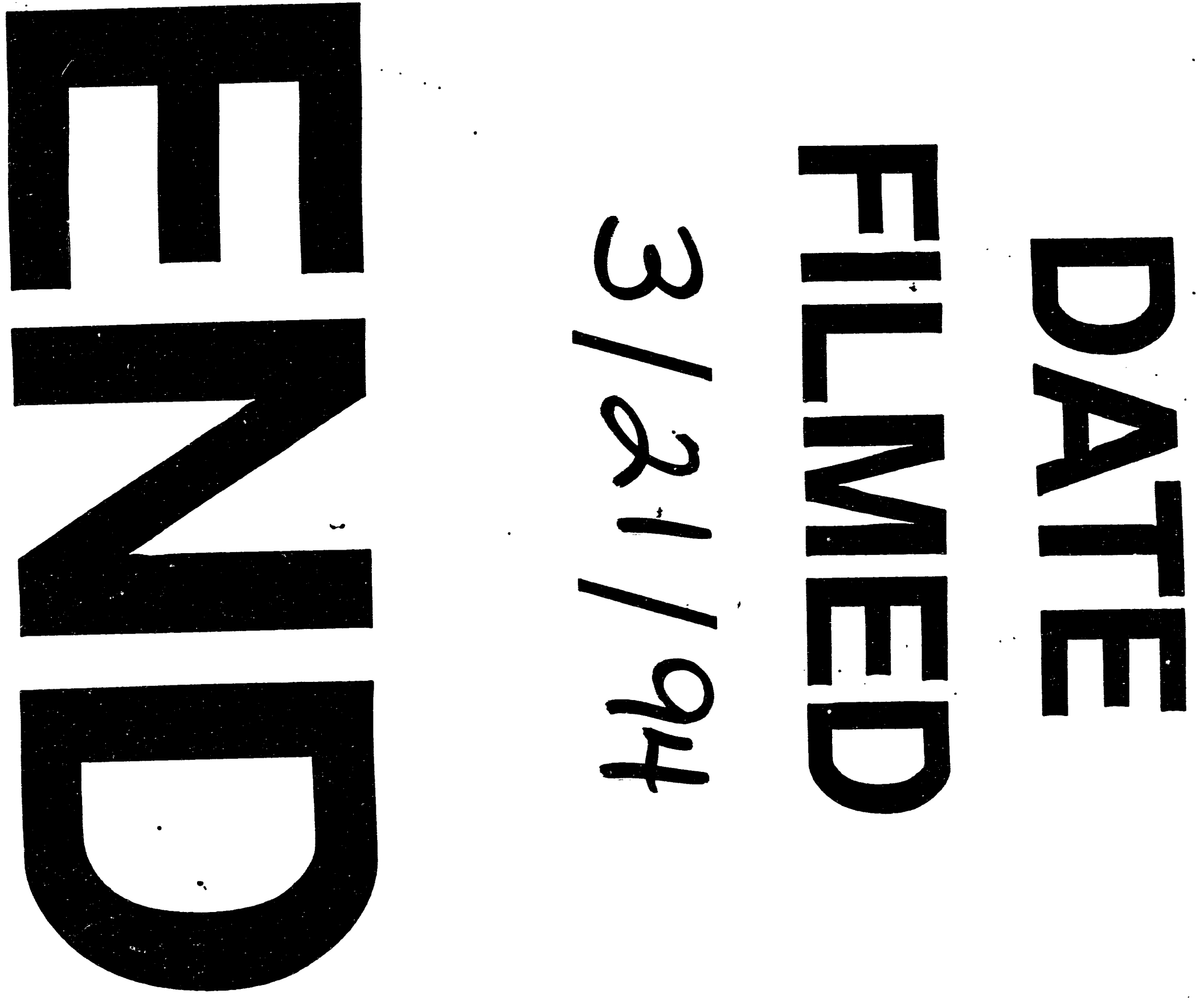
